\title{
ANWENDUNGEN DER FUZZY DATENANLYSE ZUR QUALITÄTSSICHERUNG
}

\author{
Manfred Fochem, MIT GmbH Aachen \\ Richard Weber, MIT GmbH Aachen
}

\begin{abstract}
Die Qualitätssicherung stellt eine wesentliche Aufgabe in Unternehmen des produzierenden Gewerbes dar. Ziel der entsprechenden Tätigkeiten ist es, eine gleichbleibend hohe Qualität der hergestellten Produkte bei möglichst wirtschaftlicher vorgehensweise sicherzustellen. Aus diesem Grund werden in zunehmendem Maße CAQ-Konzepte zur Automatisierung der Qualitätssicherung eingesetzt (2). Insbesondere die Auswertung von Prüfdaten kann durch entsprechende Methoden und Konzepte unterstützt werden. In diesem Beitrag wird ein Ansatz zur akustischen Qualitätskontrolle vorgestellt, wobei die Prüfung von Glas-, Porzellan- und Keramikprodukten im Vordergrund steht. Bei der akustischen Prüfung von Keramikfliesen werden diese angeschlagen und das dabei entstandene Geräusch beurteilt. Damit lassen sich bestimmte Fehler aufdecken. Eine Automatisierung dieses Vorgangs scheiterte bislang oft daran, daß die verschiedenen Fehlerlilassen sich nicht eindeutig voneinander trennen lassen und daher oft falsche Zuordrungen vorgenommen wurden. Mit Methoden der Fuzzy Datenanalyse läßt sich eine dem Problem angemessenere Beurteilung von Fliesen vornehmen [1] (3).

Es wird ein mögliches system zur Automatisierung der akustischen qualitätskontrolle vorgestellt. Die von einer angeschlagenen Fliese ausgehenden schallmuster werden über einen A/D-Wandler als Zahlenwerte im Rechner erfaßt. Dieses Zeitsignal wird durch eine Fourier-Transformation in das entsprechende Frequenzspektrum umgewandelt. Daraus werden charakteristische Merkmale zur Beurteilung der Fliesen abgeleitet.

Die an einem Versuchsaufbau erzielten Ergebnisse deuten auf die Leistungsfähigkeit der verwendeten Fuzzy Methoden hin.
\end{abstract}

\section{Literatur}

[1] Angstenberger, J., Lieven, K., Weber, R., Zimmermann, H.-J.: Fuzzy Technologien Prinzipien, Werkzeuge, Potentiale. Düsseldorf, 1993.

[2] Bläsing, J.P.: CAQ - Qualitätssicherung unter CIM-Zielen. Braunschweig, Wiesbaden, 1990 .

(3) Zimmermann, H.-J.: Fuzzy Set Theory - And Its Applications, 2nd, rev. ed., Boston, 1991. 